\title{
HP-CagA+ Regulates the Expression of CDK4/CyclinD1 via reg3 to Change Cell Cycle and Promote Cell Proliferation
}

\author{
Bin Liu ${ }^{1,2,3,+}$, Xiaokang Li ${ }^{4,+}$, Fuze Sun ${ }^{5,+}$, Xiaoling Tong ${ }^{5} \oplus$, Yanmin Bai ${ }^{5}$, Kairang Jin ${ }^{6}$, \\ Lin Liu ${ }^{6}$, Fangyin Dai ${ }^{5}$ (D) and Niannian $\mathrm{Li}^{5,6}$,* \\ 1 Teda Institute of Biological Sciences \& Biotechnology, Nankai University, Tianjin 300457, China; \\ Liubin123jinjin@sina.com \\ 2 The Key Laboratory of Molecular Microbiology \& Technology, Ministry of Education, Nankai University, \\ Tianjin 300071, China \\ 3 Tianjin Key Laboratory of Microbial Functional Genomics, Nankai University, Tianjin 300457, China \\ 4 School of Electronics and Information, Shanghai Electric Power University, Shanghai 200090, China; \\ 20162168@mail.shiep.edu.cn \\ 5 State Key Laboratory of Silkworm Genome Biology, Key Laboratory of Sericultural Biology and Genetic \\ Breeding, Ministry of Agriculture, College of Biotechnology, Southwest University, Chongqing 400715, \\ China; sunfuze@swu.edu.cn (F.S.); xltong@swu.edu.cn (X.T.); baibai666@email.swu.edu.cn (Y.B.); \\ fydai@swu.edu.cn (F.D.) \\ 6 School of Life Sciences, Nankai University, Tianjin 300071, China; kairangjin@163.com (K.J.); \\ liulin@nankai.edu.cn (L.L.) \\ * Correspondence: liniannian123liu@163.com \\ + These authors contributed equally to this work.
}

Received: 29 October 2019; Accepted: 21 December 2019; Published: 28 December 2019

\begin{abstract}
Previous studies have shown that regeneration gene 3 (reg3) is significantly expressed in gastric mucosa tissues with Helicobacter pylori (HP) cytotoxin-associated gene A (CagA)-positive (HP-CagA+). CagA-positive HP increases the risk of gastric cancer. The purpose of this study was to investigate the correlation between reg3 and HP-CagA+ and explore the effects of reg3 on the proliferation of gastric cancer cells and the development of tissues and organs. We analyzed the expression of reg3 in human tissues and organs. The results showed that reg3 expression in gastric tissues was significantly higher than that in other tissues and organs. In addition, reg3 influenced the prognosis of gastric, lung, and ovarian cancers. Immunohistochemical analysis indicated that the expression of reg3 and CagA in cancerous tissues was higher than that in adjacent tissues. $\mathrm{HP}-\mathrm{Cag} \mathrm{A}^{+}$infection of gastric cancer cells promotes reg3 expression, suggesting that reg3 may be a target gene of CagA in gastric cancer, which together affects the formation and development of gastric cancer. reg3 and CagA promote cell proliferation, and then affect the development of mouse tissues and organs by regulating G1/S phase transition of the cell cycle via the formation of the cell cycle-dependent complex CDK4/CyclinD1. This is the first study that shows the influence of CagA on the cell cycle and induction of cell proliferation by promoting reg3 expression.
\end{abstract}

Keywords: gastric cancer; reg3; HP-CagA+; cell cycle; CDK4/CyclinD1

\section{Introduction}

Gastric cancer is one of the most common malignant tumors in the world. The five-year survival rate is less than $40 \%$, thereby making it a serious threat to human health [1]. At present, there is still no effective treatment for gastric cancer [2]. Therefore, it is necessary to further explore the mechanism of the occurrence and development of gastric cancer, and to prevent the incidence of gastric 
cancer. The potential role of Reg in tumors, particularly in digestive system tumors, has been recently investigated [3,4]. It is currently determined that the Reg family proteins belong to the C-type lectin family, depending on the sequence characteristics of the protein [3]. These were estimated to have about 120 amino acids constituting a calcium-dependent carbohydrate recognition domain (CRD) [5], which are present in serum [6], extracellular matrix [7], and cell membrane [8], and C-type lectin on the cell membrane can participate in swallowing, cell adhesion, or humoral immune response $[9,10]$.

Studies have shown that Helicobacter pylori infection is an important cause of gastric cancer [11,12]. Epidemiology shows that the major protein toxic factors carried by $H$. pylori strains is CagA, which can increase the risk of gastric cancer [13,14]. In human gastric mucosa tissues, the expression of Reg3 was significantly increased in the presence of CagA compared to CagA-negative H. pylori-infected individuals [15]. RT-PCR (reverse transcription-polymerase chain reaction) demonstrated a significant increase in the expression of rat reg3 in CagA-positive HP-infected gastric mucosal biopsies but not in CagA-negative tissues [16]. reg3 may act as a targeting molecule for CagA in gastric epithelial cells $[15,16]$. CagA-mediated SHP signaling can lead to abnormalities in epithelial cell polarity $[17,18]$. In particular, the transformation of potentially carcinogenic epithelial cells into mesenchymal cells (EMT) is attributed thereto [19]. Studies involving transgenic mice have further supported the hypothesis that CagA may be a potential carcinogenic factor [20]. However, how reg3 interacts with CagA in the stomach and affects the occurrence and development of gastric cancer, as well as how to regulate downstream genes to induce tumors, have not been studied to date. Thus, the purpose of this study was to explore the carcinogenic mechanism of Reg3 and CagA and to provide some basis for cancer treatment.

\section{Results}

\section{1. reg3 Is Highly Expressed in Gastric Cancer and Affects the Prognosis of Patients}

reg3 is a member of the regenerative gene family and has attracted much attention in relation to inflammatory wounds and digestive tract tumors. The expression of reg3 in three common tumors, including gastric, lung, and ovarian cancer, and the effect of the reg3 on survival and prognosis of patients were analyzed using TCGA data (Figure 1A,B). The analysis showed that the expression level of reg3 in these three tumor tissues was higher than that in normal tissues. In addition, the upregulation of this gene is associated with poor prognosis. Meanwhile, the human protein database was first used to analyze the expression of reg3 in human tissues and organs (www.proteinatlas.org) (Figure 1C). The results indicated that the level of reg3 expression in gastric tissues, including the stomach, duodenum, and small intestine, was relatively higher than other the tissues and organs. reg3 is closely related to the occurrence of gastric cancer, and is an important growth gene and regulatory target in gastric cancer pathogenesis. The results of our analysis prove that reg3 plays an important role in the development of the stomach and influences the formation and development of gastric cancer. 

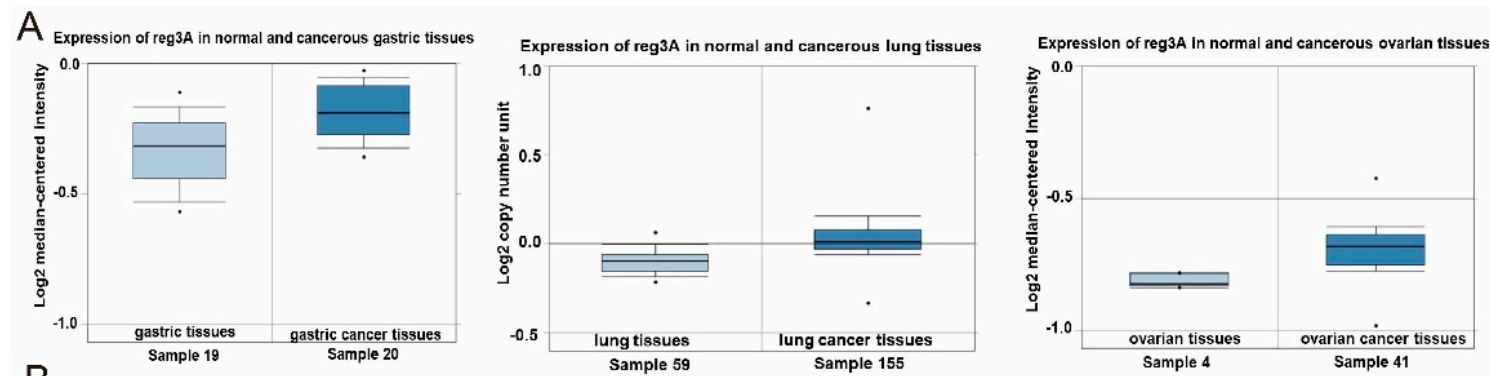

B
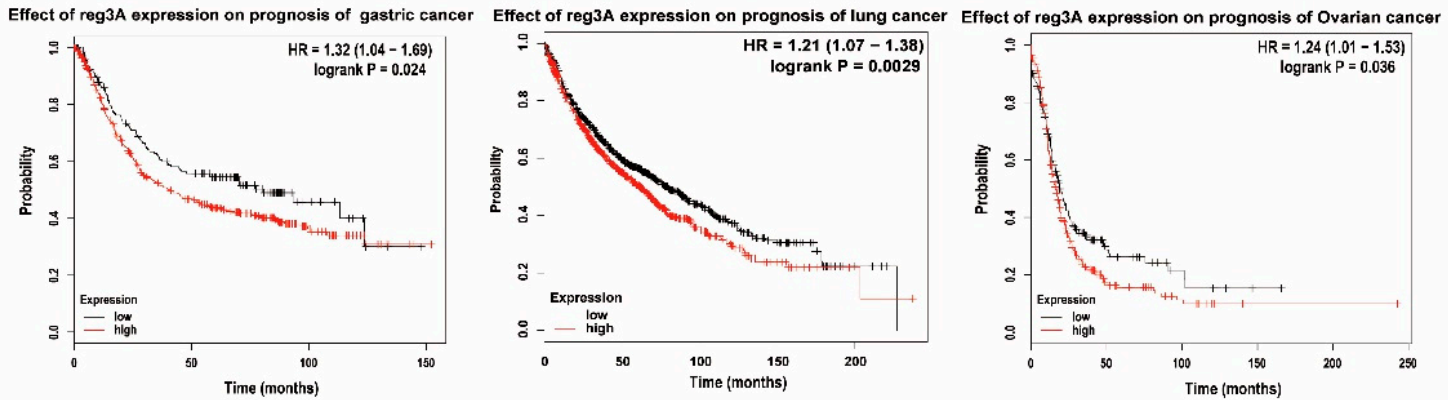

C
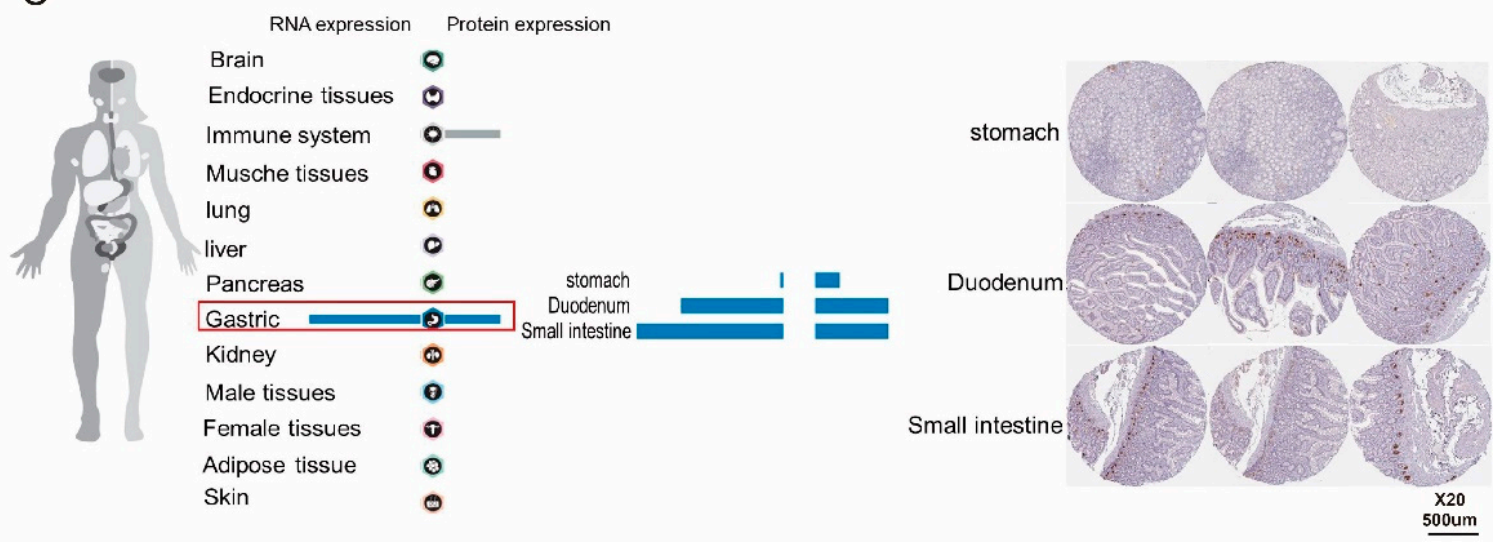

Figure 1. reg3 is highly expressed in gastric cancer and affects the prognosis of patients. (A). The expression of reg3 in three common tumors, including gastric, lung, and ovarian cancer. (B). the effect of the reg3 on survival and prognosis of patients. (C). the expression of reg3 in human tissues and organs.

\subsection{CagA Promotes Cell Proliferation by Regulating reg3}

Helicobacter pylori (HP) infection is an important cause of gastric cancer. The CagA carried by the $\mathrm{HP}$ can increase the risk of gastric cancer. Immunohistochemistry was used to detect the expression of CagA and reg3 in gastric cancer (Figure 2A,B). The results show that the expression level of CagA and reg3 in cancerous tissues is higher than that in the adjacent tissues. Reg3 was upregulated in gastric cancer cell lines, and the effect of Reg3 on cell proliferation was detected by the MTT assay and cell counting (Figure 2C). The results show that the upregulation of reg3 can promote cell proliferation. In addition, CagA-positive H. pylori $\left(\mathrm{HP}^{-} \mathrm{CagA}^{+}\right)$was used to infect gastric cancer cells to detect changes in cell proliferation (Figure 2D). The results show that as infection time is prolonged, cell proliferation is accelerated. RT-PCR analysis indicated that the expression of reg3 in gastric mucosa infected by CagA-positive H. pylori significantly increased in mice while the expression of Reg3 in gastric mucosa infected by CagA-negative H. pylori was comparable to that in normal gastric mucosa. In this study, $\mathrm{CagA}$ and Reg3 were upregulated in gastric cancer tissues and promote gastric cancer cell proliferation. However, the relationship between CagA and Reg3 expression is unclear. After HP-CagA ${ }^{+}$was transfected into gastric cancer cells, reg3 expression was detected at 0, 24, and $48 \mathrm{~h}$ (Figure 2E,F). The experimental results show that the expression of reg3 gradually increased with the prolongation of HP-CagA ${ }^{+}$infection time. This indicates that CagA can promote the expression of reg3. 
A Expression and distribution of cagA in gastric cancer patients
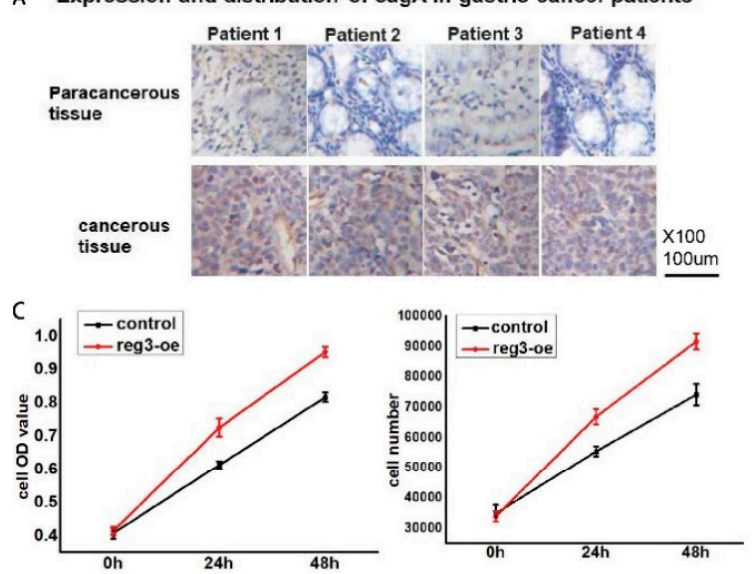

B Expression and distribution of reg3 in gastric cancer patients
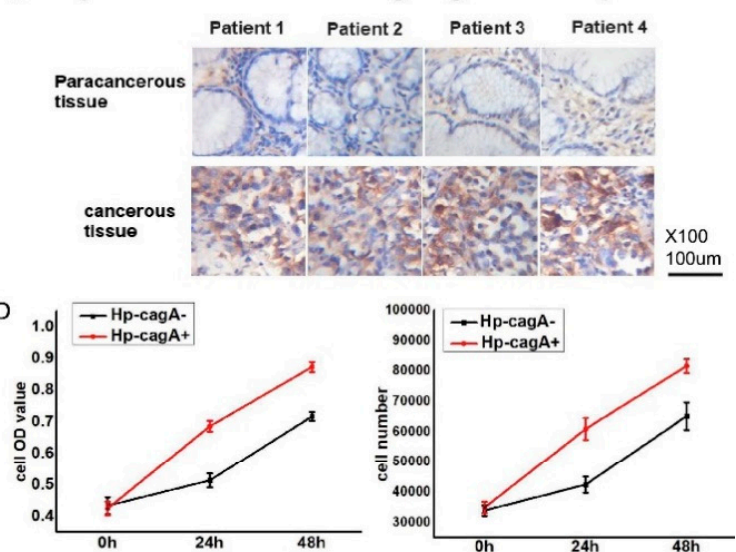

E

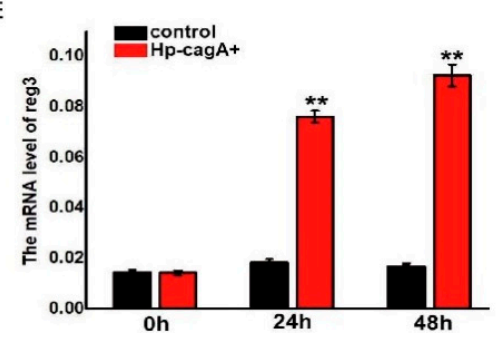

$\mathbf{F}$

Figure 2. CagA promotes cell proliferation by regulating reg3. (A). Immunohistochemistry was used to detect the expression of CagA in gastric cancer. (B). Immunohistochemistry was used to detect the expression of reg3 in gastric cancer. (C). The effect of reg3 on cell proliferation was detected by the MTT assay and cell counting. (D). The effect of CagA on cell proliferation was detected by the MTT assay and cell counting. (E). The mRNA level of reg3 expression was detected at 0,24 , and $48 \mathrm{~h}$ after $\mathrm{HP}-\mathrm{CagA}^{+}$was transfected into gastric cancer cells. ${ }^{* *} p$ value $\leq 0.01$. (F). The protein level of reg3 expression was detected at 0,24 , and $48 \mathrm{~h}$ after ${\mathrm{HP}-C a g A^{+}}^{+}$was transfected into gastric cancer cells.

\section{3. reg3 Regulates the Growth and Development of Tissues and Organs}

The above experiments proved that reg3 can promote cell proliferation (Figure 2C). To explore whether reg3 could affect the growth of mice, we constructed an adeno-associated virus overexpressing reg3 (AAV-Reg3-oe) (Figure 3A). Approximately eight weeks after AAV-Reg3-oe injection into the tail vein, the expression of reg3 was assessed using a small animal imaging instrument (Figure 3B). The results showed that reg3 was expressed systemically. The mice were then dissected, and the growth of the liver, lungs, and kidneys was assessed (Figure 3C-E). When reg3 was overexpressed, the volume and weight of the liver, lung, and kidney were higher than those of the control group. These results agree with our conclusion that reg3 promotes cell proliferation, i.e., reg3 promotes the growth of tissues and organs by inducing cell proliferation. The level of $r e g 3$ expression in each tissue and organ was assessed, and that of the reg3-oe group was determined to be upregulated (Figure 3F,G). 


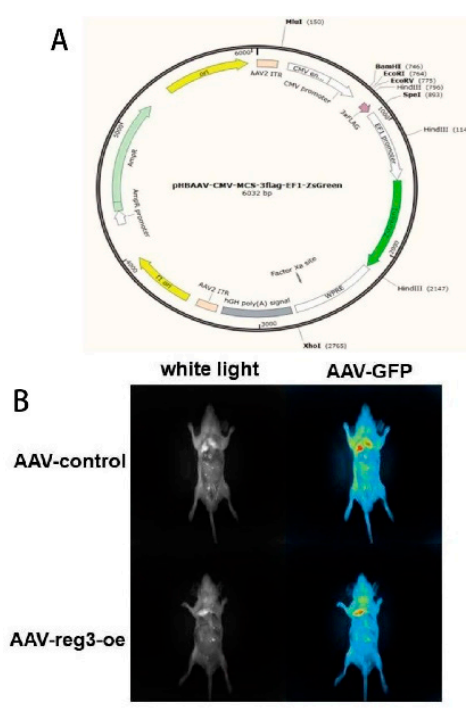

$\mathbf{F}$

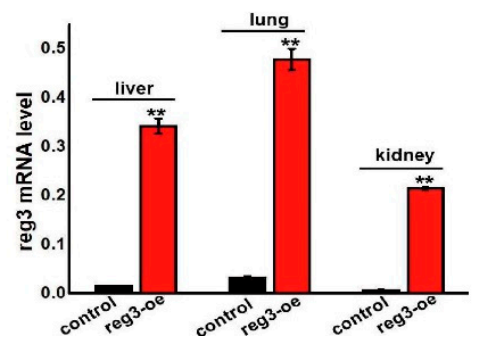

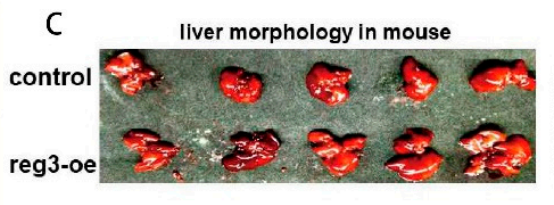
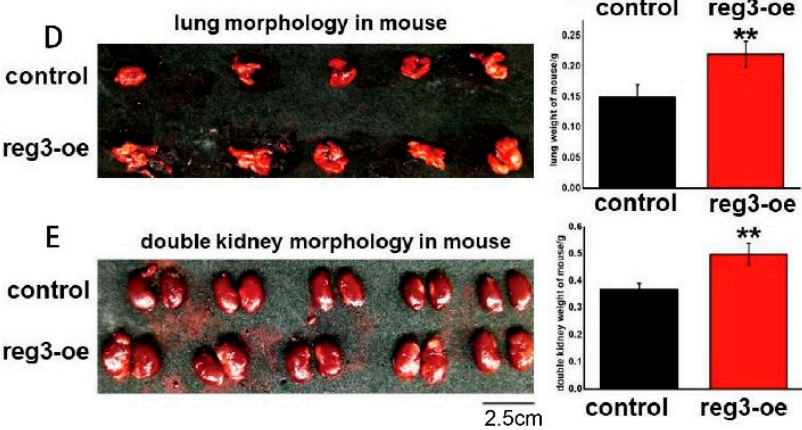

G

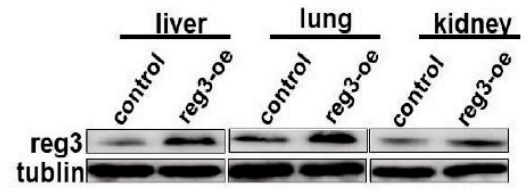

Figure 3. reg3 regulates the growth and development of tissues and organs. (A). The viral vector map of adeno-associated virus overexpressing reg3 (AAV-Reg3-oe). (B). The expression of reg3 was assessed using a small animal imaging instrument. (C). The mice were dissected, and the growth of the liver was assessed. (D). The mice were dissected, and the growth of the lungs was assessed. (E). The mice were dissected, and the growth of the kidneys was assessed. (F). The mRNA level of reg3 in each tissue and organ was assessed. (G). The protein level of reg3 in each tissue and organ was assessed. ** $p$ value $\leq 0.01$.

\subsection{CagA and reg3 Alter the Cell Cycle}

The above experiments demonstrate that CagA promotes the expression of reg3 and stimulates cell proliferation with Reg3 (Figure 2C-F). To explore the causes of CagA and reg3 promoting cell proliferation, cell cycle changes were assessed after the upregulation of reg3 in gastric cancer cells and H. pylori infection of gastric cancer cells (Figure 4A). The results showed that the proportion of G1 phase gradually decreased with the prolongation of reg3 overexpression. This also implies that reg3 promotes the progression of the cell cycle. At the same time, $\mathrm{HP}^{-C a g A^{+}}$was used to infect gastric cancer cells to detect cell cycle changes (Figure 4B). The results show that the proportion of G1 phase gradually decreased with the prolongation of infection. This indicates that CagA promotes the progression of the cell cycle. Combined with the above experiments, we conclude that CagA and reg3 promote cell proliferation by altering the cell cycle. Of course, the reason for the slowdown of cell proliferation is not only the change of the cell cycle, but also the increase of the proportion of apoptosis. Therefore, the effects of reg3 and CagA on apoptosis were examined (Supplement Figures S1 and S2). It was found that $r e g 3$ and $C a g A$ can inhibit the apoptosis of gastric cancer cells. This article mainly analyzes changes in the cell cycle, so it does not detail the mechanism of reg3 and $\mathrm{CagA}$ affecting apoptosis. 

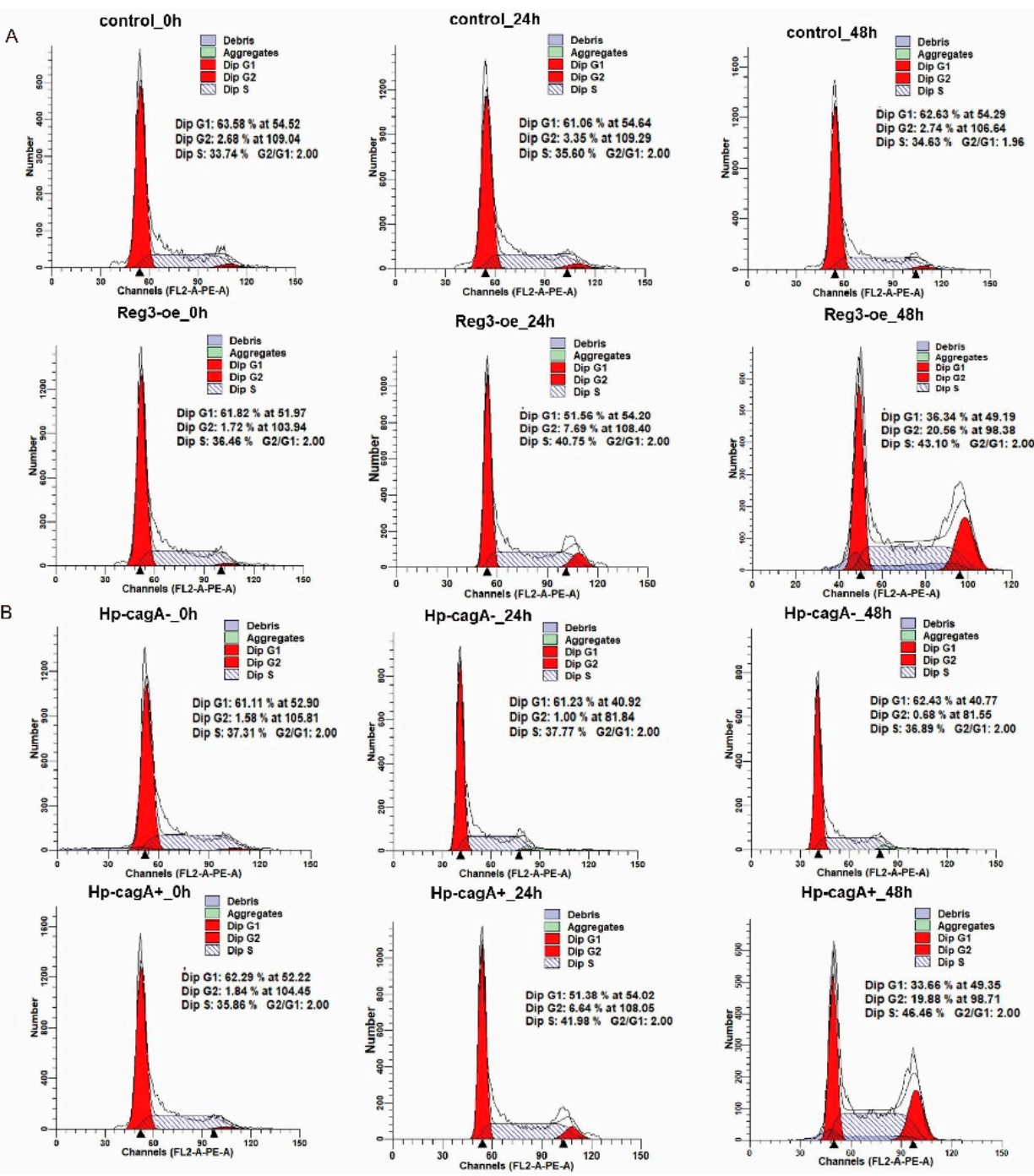

Figure 4. CagA and reg3 alter the cell cycle. (A). cell cycle changes were assessed after the upregulation of reg3 in gastric cancer cells. (B). cell cycle changes were assessed after $H$. pylori infection of gastric cancer cells.

\subsection{CagA and reg3 Regulate the Expression of CDK4 and CyclinD1}

Both reg3 and CagA can affect the transition from G1 to S phase of the cell cycle (Figure 4). Key to the transition from $\mathrm{G} 1$ to $\mathrm{S}$ phase is the formation of cell cycle-dependent complex CDK4/cyclinD1 (Figure 5A). However, the regulatory mechanisms of CagA and reg3 in relation to cell cycle progression are unclear. Changes in cell cycle-dependent genes $C D K 4$ and cyclinD1 were detected after upregulation of reg3 in gastric cancer cells. The results show that reg3 can promote the expression of CDK4 and cyclinD1 (Figure 5B,D). Simultaneously, changes in the expression of CDK4 and cyclinD1 were detected after HP-CagA+ infection of gastric cancer cells (Figure 5C,D). The results show that CagA can promote the expression of CDK4 and CyclinD1. The formation of the cell cycle-dependent complex CDK4/CyclinD1 is key to the transition from cell cycle G1 to S phase. Therefore, the effect of Reg 3 on the expression and distribution of CDK4 and CyclinD1 were assessed by immunofluorescence (Figure 5E). The results showed that overexpression of reg3 increased the distribution of CDK4 and cyclinD1 in the nucleus, and co-localization was observed. Immunofluorescence was also used to detect the effects of HP-CagA+-infected cells on the expression and distribution of CDK4 and CyclinD1 (Figure 5F). The results showed that HP-CagA+ infection increased the distribution of CDK4 and CyclinD1 in 
the nucleus, and co-localization was observed. This may be the mechanism by which reg3 and CagA regulate the cell cycle.

A

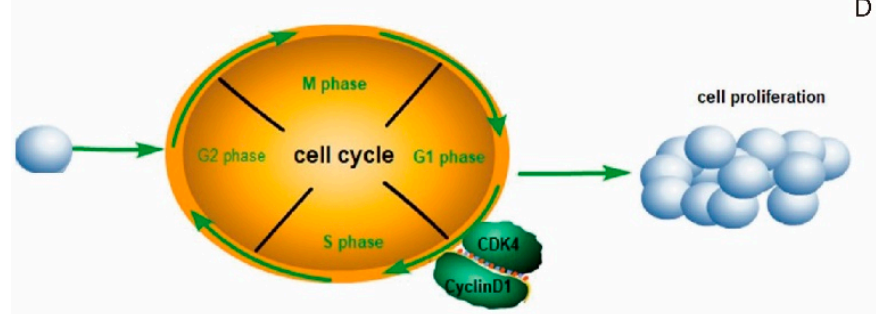

D

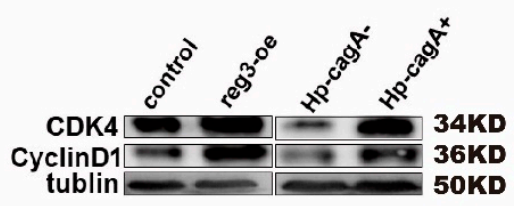

B

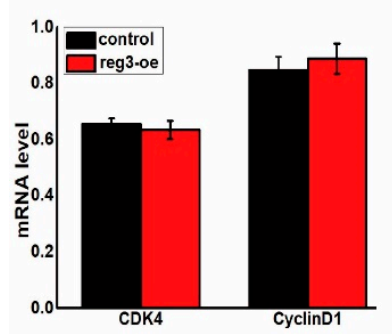

C

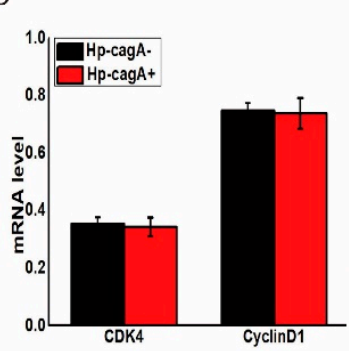

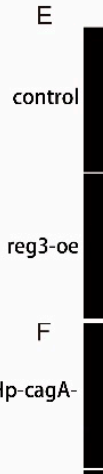

DAPI tublin 
and co-localization was weaker. These results suggest that reg3 may be a key gene for CagA to alter the cell cycle and promote cell proliferation. This may also be the regulatory mechanism of $H$. pylori in promoting gastric cancer.
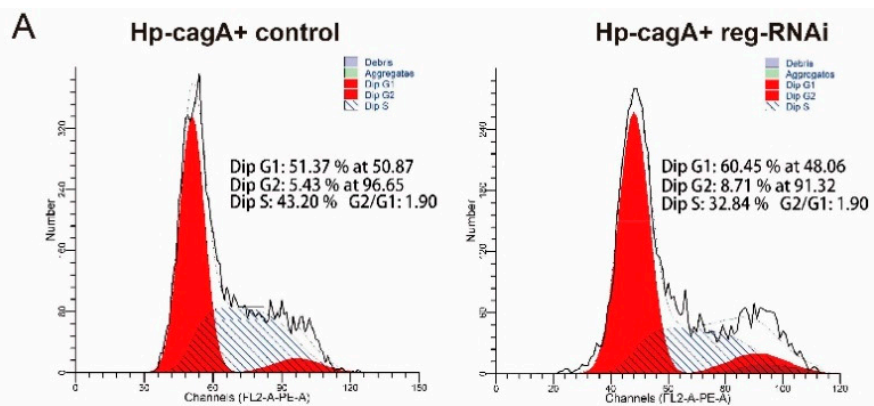

Hp-cagA+ reg-oe
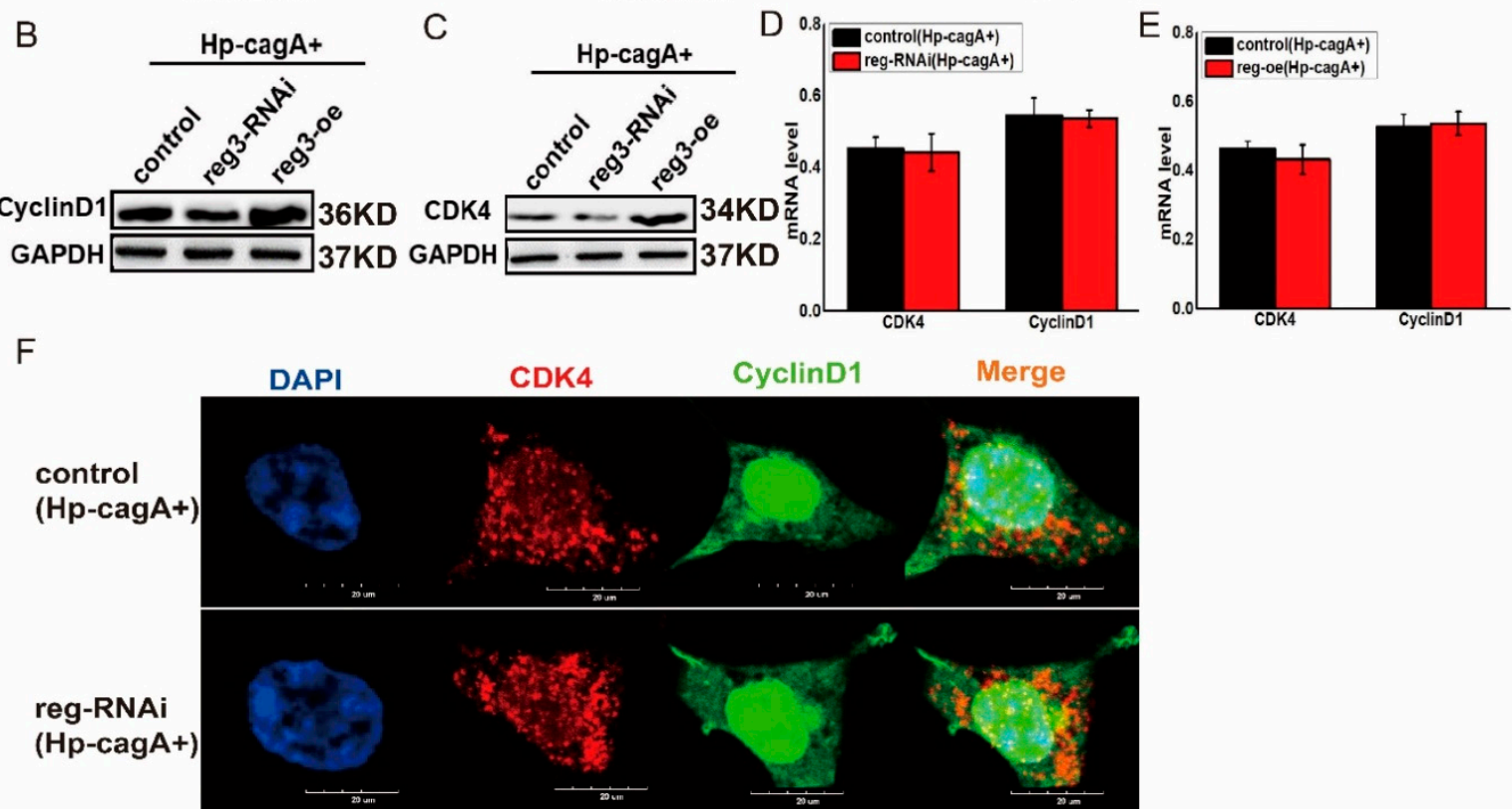

Figure 6. CagA and reg3 regulate the expression of CDK4 and CyclinD1. (A). In HP-CagA+-infected gastric cancer cells, the cell cycle was detected after downregulation and upregulation of reg3. (B). In HP-CagA+-infected gastric cancer cells, the expression of CDK4 was detected when reg3 expression was altered. (C). In HP-CagA+-infected gastric cancer cells, the expression of Cyclin D1 was detected when reg3 expression was altered. (D). The mRNA level of CDK4/CyclinD1 were examined after downregulation of reg3 expression in HP-CagA+-infected gastric cancer cells. (E). The mRNA level of CDK4/CyclinD1 were examined after upregulation of reg3 expression in HP-CagA+-infected gastric cancer cells. (F). Immunofluorescence was used to detect the effects of HP-CagA+ and reg3 on the expression and distribution of CDK4 and cyclinD1.

\section{Discussion}

The occurrence, development, and invasion of gastric cancer is a complex and continuous process. Most people with $H$. pylori infection are asymptomatic. Chronic gastritis and other diseases have occurred in only $10 \%$ of infected people [21]. However, such diseases caused by $H$. pylori infection do increase the risk of stomach cancer. Based on this evidence, the World Health Organization's International Cancer Research Agency classified Helicobacter pylori as a Class I carcinogen in 1994 [22]. Studies have shown that cagA-positive H. pylori infection has an important impact on the occurrence and development of gastric cancer $[11,23]$. Reg3 expression influences the occurrence and development of gastric cancer [3]. In this study, immunohistochemistry was used to detect the expression of CagA and reg3 in gastric cancer and adjacent normal tissues (Figure 2A,B). We found that CagA and reg3 were 
upregulated in gastric cancer tissues and downregulated in the adjacent normal tissues. We concluded that CagA and reg3 play an important role in the occurrence and development of gastric cancer, and Reg3 may interact with CagA to influence the formation and development of gastric cancer. In gastric cancer cell lines, RT-PCR and Western blotting showed that the expression of reg3 significantly increased in CagA-positive H. pylori-infected gastric cancer cells but not in CagA-negative gastric cancer cells (Figure 2E,F). These findings suggest that reg3 may be a target molecule of CagA in gastric cancer cells.

This study has also shown that CagA and reg3 can promote cell proliferation by affecting the cell cycle (Figure 4). We also wanted to elucidate how CagA and reg3 regulate the cell cycle and cell proliferation. We found that CagA and reg3 could affect the formation of the cell cycle-dependent complex, CDK4/CyclinD1 (Figure 5), which may be responsible for changes in the expression of cagA and reg3 during the G1/S transition of the cell cycle. At the same time, we found that the regulation of the cell cycle by CagA was weaker after reg3 was downregulated (Figure 6). These findings indicate that reg3 may be a target for CagA to regulate the cell cycle.

In summary, based on the limited number of studies on reg3 in gastric cancer, this study elucidated the mechanism by which CagA-positive H. pylori can regulate the cell cycle through reg3, and ultimately promote the formation and development of tumors (Figure 7).

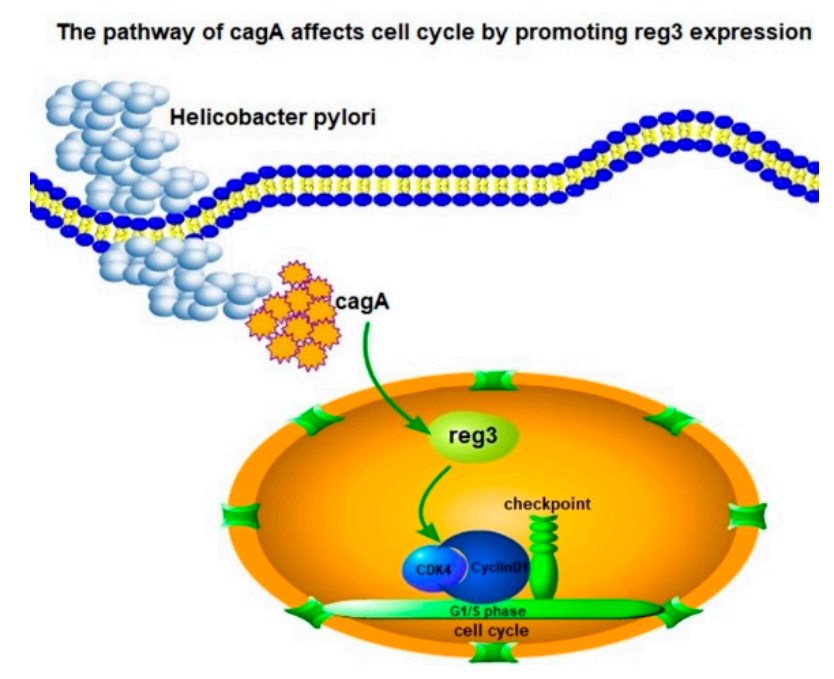

Figure 7. reg3 regulates cell cycle after CagA-positive H. pylori infection.

Although H. pylori is considered to be a class of carcinogens, it may not be a direct mutagen. CagA and its related pathogenicity island (CagPAI) are associated with increased cancer risk. CagA is transported to host cells through the type IV secretion system encoded by CagPAI, localized to the plasma membrane, and tyrosine phosphorylated by a variety of Src kinases. Tyrosine phosphorylated CagA activates SHP-2, which enhances cell proliferation. The expression of cyclin D1 induced by H. pylori is CagPAI dependent. It was demonstrated in this paper that CagA can regulate cyclin D1 expression through reg3, thereby affecting cell proliferation. Then, CagA's regulation of reg3 is also cagPAI dependent. This is the question we want to study next. Generally, our research results may serve as a theoretical basis for the clinical treatment of gastric cancer.

\section{Materials and Methods}

\subsection{Experimental Materials}

Cancer tissues and corresponding adjacent tissues of gastric cancer were provided by the Military Medical University. The gastric cancer cell line MKN45 was provided by the Army Medical University. 
Anti-Reg3 (ab202057), anti-tubulin (ab18251), anti-CDK4 (ab108357), anti-CagA (ab224836), and anti-cyclin D1 (ab16663) were purchased from Abcam.

\subsection{Hp-CagA Infection in Human Gastric Cancer Cells}

Actively proliferating cells were plated at $2.5 \times 10^{5}$ cells per well in a 6-well plate. When the cells are attached, replace the cell culture medium without antibiotics. The inoculating ring was used to scrape live H. pylori bacteria from the culture medium, suspended in phosphate-buffered saline (PBS), adjusted the bacterial density, and added the bacterial solution to the cell culture dish at a ratio of 1:100 bacteria to cells, and continued the culture. The statement of Hp strains: ATCC No. 43504; Application: Media testing Quality control strain Susceptibility testing Quality control strain for API products Enteric Research; Type Strain; Biosafety Level 2, Biosafety classification is based on U.S. Public Health Service Guidelines, it is the responsibility of the customer to ensure that their facilities comply with biosafety regulations for their own country.

\subsection{Adeno-Related Carriers, Packaging Cells, and Strains}

Virus packaging system: Three plasmids system, pAAV RC, helpers, and shuttle plasmids (carrying the target gene or shRNA). Packaging cell line: aav-293 cells, long-term preservation by hanheng biological company, culture conditions are DMEM $+10 \% \mathrm{FBS}, 37^{\circ} \mathrm{C}, 5 \% \mathrm{CO}_{2}$, relative humidity $95 \%$. Strain: E. coli strain stbl3, used to amplify adeno-associated vector and auxiliary packaging vector plasmid.

\subsection{Gene Expression Analysis}

We used UALCAN (http://ualcan.path.uab.edu) to detect reg3 expression in different tumor tissues and their corresponding normal tissues, and to compare changes in expression levels of reg3. UALCAN allows scientists to gain access to terabytes of data and close to 1000 reports. To systematize analyses from The Cancer Genome Atlas (TCGA) pilot and to scale their execution to the dozens of remaining diseases to be studied, UALCAN now sits atop $\sim 40$ terabytes of TCGA data and reliably executes more than 6000 pipelines per month.

\subsection{Immunohistochemistry}

Immunohistochemical steps: (1) Baking sheet: The tissue chips were placed in a $63{ }^{\circ} \mathrm{C}$ oven for $1 \mathrm{~h}$. (2) Dewaxing: After baking, the tissues were placed in an automatic dyeing machine for dewaxing; (3) Antigen repair: The tissues were rinsed with pure water thrice, and then immersed in citric acid repair solution or EDTA repair solution on an electromagnetic oven and heated; (4) EDTA thermal repair: the tissues were boiled for $20 \mathrm{~min}$. (5) Blocking: Commercialized ready-to-use blockers were used, and the tissues were placed onto tablets for 10 to $15 \mathrm{~min}$. (6) The primary and secondary antibodies were added sequentially. (7) DAB color development: Diluted DAB was added onto the tablet and the intensity of the resulting color was assessed. (8) The tissues were stained with hematoxylin and then sealed.

\subsection{RNA Extraction and $C D N A$ Synthesis}

Total RNA was extracted from cells using a MicroElute Total RNA kit (Omega Bio-Tek, Norcross, GA, USA). RNA from organ/tissues was extracted using TRIzol reagent (Invitrogen, USA) according to the manufacturer's instructions. RNA quality was verified spectrophotometrically with an A260/A280 ratio between 1.8 and 2.0. cDNA was synthesized from $2 \mu \mathrm{g}$ DNase-treated total RNA samples with oligo(dT) primers and PrimeScript ${ }^{\mathrm{TM}}$ reverse transcriptase (TaKaRa) according to the manufacturer's instructions. cDNAs were diluted 10 times and used in qRT-RCR. 


\subsection{Fluorescence $q R T-P C R$}

Fluorescence qRT-PCR experiments were performed on a quantitative real-time polymerase chain reaction detection system (CFX96, BIO-RAD, Hercules, CA, USA) with SYBR Premix EX Taq kit (Takara, Dalian, China), using the recommendations of the manufacturer. The primers for RT-PCR in this study were designed by Primer 5.0 and are as follows:

reg3-s: GTGACTCCTGATTGCCTCCTC, reg3-a: GCTACTCCACTCCCAACCTTC, CDK4-s: ATGGCACTTACACCCGTGGTT, CDK4-a: CTGGTCGGCTTCAGAGTTTCC, CyclinD1-s: AAGATCGTCGCCACCTGGATG, CyclinD1-a: GATGGAGTTGTCGGTGTAGATGCA, GAPDH-s: TGACTTCAACAGCGACACCCA, GAPDH-a: CACCCTGTTGCTGTAGCCAAA.

\subsection{Cell Proliferation and Cell Cycle Test}

The cell line of MKN45 was supplied by The Army Military Medical University (Chongqing, China). When the cell paved rate of more than $70 \%$, transfected with plasmid, including empty vector (transfection reagent lip2000 come from Roche). The cells were preserved after transfection for 0, 24, 48 , and $72 \mathrm{~h}$, and changes in cell number and cell cycle were assessed by flow cytometry.

The MTT cytotoxicity assay was used to assess cell viability and proliferation [24]. At $48 \mathrm{~h}$ post-transfection, a cell suspension was prepared and loaded into 96-well culture plates. Approximately $10 \mu \mathrm{L}$ of the MTT reagent (Beyotime Biotechnology) were added to each well and the plates were incubated for $4 \mathrm{~h}$. Then, the plate cover was removed, and the absorbance of each well and the blank was measured at a wavelength of $570 \mathrm{~nm}$ using a microtiter plate reader.

\subsection{Immunofluorescence}

Changes in the distribution of Reg3 were determined by immunofluorescence using the following primary antibodies: Rabbit anti-Reg3 (Cell Signaling Technology). Nuclei were stained with DAPI (Beyotime Biotechnology). Fluorescent-conjugated secondary antibodies were obtained from Beyotime Biotechnology, Inc. Confocal images were collected using an Olympus TCS SP5 confocal microscope with $40 \times / 1.25$ oil objectives.

Supplementary Materials: The following are available online at http:/www.mdpi.com/1422-0067/21/1/224/s1.

Author Contributions: B.L. and N.L. participated in the design of the article ideas and completed some experiments. X.L. and F.S. analyzed the experimental data and visualized the figures. X.T. and Y.B. drafted the original article. K.J. and L.L. participated in the revision of the article. F.D. provided experimental materials and financial support for the article. All authors have read and agreed to the published version of the manuscript.

Funding: The research was funded by the Startup Foundation for Junior Faculty, Nankai University (No. 63191439) and the National Natural Science Foundation of China (No. 31830094), and Funds of China Agriculture Research System (No. CARS-18-ZJ0102).

Conflicts of Interest: The authors declare no conflict of interest.

\section{References}

1. Ichiyasu, N.; Tada, S.; Kamio, T.; Ueno, N.; Seki, T.; Suko, H. Malignant gastrointestinal stromal tumor of the duodenum after gastrectomy due to early gastric cancer. Report of a case. Jpn. J. Gastro-Enterol. 1998, 95, 1235-1239.

2. Takahashi, T.; Saikawa, Y.; Igarashi, T.; Tsuwano, S.; Kumagai, K.; Nakamura, R.; Ooyama, T.; Wada, N.; Takeuchi, H.; Takaishi, H.; et al. Octreotide acetate enables the administration of chemoradiotherapy, including the oral anticancer drug S-1, in gastric cancer patients with malignant gastrointestinal obstruction. Oncol. Lett. 2010, 1, 673-677.

3. Waldum, H.L.; Qvigstad, G.; Sandvik, A.K. Reg protein in gastric cancer tumour cells. FEBS Lett. 2003, 553, 464-465. [CrossRef] 
4. $\quad$ Li, N.; Feng, Y.; Hu, Y.; He, C.; Xie, C.; Ouyang, Y.; Artim, S.C.; Huang, D.; Zhu, Y.; Luo, Z.; et al. Helicobacter pylori CagA promotes epithelial mesenchymal transition in gastric carcinogenesis via triggering oncogenic YAP pathway. J. Exp. Clin. Cancer Res. 2018, 37, 280. [CrossRef]

5. Wakita, A.; Motoyama, S.; Sato, Y.; Koyota, S.; Usami, S.; Yoshino, K.; Sasaki, T.; Imai, K.; Saito, H.; Minamiya, Y. REG Ialpha activates c-Jun through MAPK pathways to enhance the radiosensitivity of squamous esophageal cancer cells. Tumour Biol. 2015, 36, 5249-5254. [CrossRef]

6. Mitani, Y.; Oue, N.; Matsumura, S.; Yoshida, K.; Noguchi, T.; Ito, M.; Tanaka, S.; Kuniyasu, H.; Kamata, N.; Yasui, W. Reg IV is a serum biomarker for gastric cancer patients and predicts response to 5-fluorouracil-based chemotherapy. Oncogene 2007, 26, 4383-4393. [CrossRef]

7. Stejskal, D.; Karpisek, M.; Horakova, D.; Cizek, L.; Janoutova, G.; Janout, V. Serum Reg-Ialpha is not suitable marker of metabolic syndrome. Bratisl. Lek. Listy 2007, 108, 138-140. [PubMed]

8. Cui, W.; Liu, J.L.; Shi, B.Y. Reg family and pancreatic islet beta cell. Sheng Li Ke Xue Jin Zhan 2009, 40, 55-58. [PubMed]

9. Aida, K.; Saitoh, S.; Nishida, Y.; Yokota, S.; Ohno, S.; Mao, X.; Akiyama, D.; Tanaka, S.; Awata, T.; Shimada, A.; et al. Distinct cell clusters touching islet cells induce islet cell replication in association with over-expression of Regenerating Gene (REG) protein in fulminant type 1 diabetes. PLoS ONE 2014, 9, e95110. [CrossRef] [PubMed]

10. Bone, A.J.; Banister, S.H.; Zhang, S. The REG gene and islet cell repair and renewal in type 1 diabetes. Adv. Exp. Med. Biol. 1997, 426, 321-327.

11. Lawniczak, M.; Starzynska, T. Helicobacter pylori CagA(+) infection in gastric cancer patients. Pol. Merkur. Lekarski 2002, 13, 216-220.

12. Saber, T.; Ghonaim, M.M.; Yousef, A.R.; Khalifa, A.; al Qurashi, H.; Shaqhan, M.; Samaha, M. Association of Helicobacter pylori cagA Gene with Gastric Cancer and Peptic Ulcer in Saudi Patients. J. Microbiol. Biotechnol. 2015, 25, 1146-1153. [CrossRef]

13. Parsonnet, J.; Friedman, G.D.; Orentreich, N.; Vogelman, H. Risk for gastric cancer in people with CagA positive or CagA negative Helicobacter pylori infection. Gut 1997, 40, 297-301. [CrossRef]

14. Sasazuki, S.; Inoue, M.; Iwasaki, M.; Otani, T.; Yamamoto, S.; Ikeda, S.; Hanaoka, T.; Tsugane, S. Japan Public Health Center Study, Effect of Helicobacter pylori infection combined with CagA and pepsinogen status on gastric cancer development among Japanese men and women: A nested case-control study. Cancer Epidemiol. Prev. Biomark. 2006, 15, 1341-1347. [CrossRef]

15. Fukui, H.; Sekikawa, A.; Chiba, T. REG (regenerating gene) protein-roles of REG protein in H. pylori-induced gastritis and gastric cancers. Nihon Rinsho Jpn. J. Clin. Med. 2005, 11 (Suppl. 63), 98-102.

16. Kang, D.W.; Hwang, W.C.; Park, M.H.; Ko, G.H.; Ha, W.S.; Kim, K.S.; Lee, Y.C.; Choi, K.Y.; Min, D.S. Rebamipide abolishes Helicobacter pylori CagA-induced phospholipase D1 expression via inhibition of NFkappaB and suppresses invasion of gastric cancer cells. Oncogene 2013, 32, 3531-3542. [CrossRef]

17. Masu, H. CagA tyrosine phosphorylation motif structure and SHP-2 binding ability of Helicobacter pylori studied in stomach cancer and duodenal ulcer cell lines. Hokkaido J. Med. Sci. 2006, 81, 121-128.

18. Tsutumi, R.; Hatakeyama, M. Helicobacter pylori CagA and SHP-2 tyrosine phosphatase. Seikagaku 2005, 77, 1269-1273.

19. Sougleri, I.S.; Papadakos, K.S.; Zadik, M.P.; Mavri-Vavagianni, M.; Mentis, A.F.; Sgouras, D.N. Helicobacter pylori CagA protein induces factors involved in the epithelial to mesenchymal transition (EMT) in infected gastric epithelial cells in an EPIYA- phosphorylation-dependent manner. FEBS J. 2016, 283, 206-220. [CrossRef]

20. Vaziri, F.; Peerayeh, S.N.; Alebouyeh, M.; Maghsoudi, N.; Azimzadeh, P.; Siadat, S.D.; Zali, M.R. Novel effects of Helicobacter pylori CagA on key genes of gastric cancer signal transduction: A comparative transfection study. Pathog. Dis. 2015, 73, ftu021. [CrossRef]

21. Chen, S.Y.; Zhang, R.G.; Duan, G.C. Pathogenic mechanisms of the oncoprotein CagA in H. pylori-induced gastric cancer (Review). Oncol. Rep. 2016, 36, 3087-3094. [CrossRef] [PubMed]

22. Al-Marhoon, M.S.; Nunn, S.; Soames, R.W. The association between cagA+ H. pylori infection and distal gastric cancer: A proposed model. Dig. Dis. Sci. 2004, 49, 1116-1122. [CrossRef] [PubMed] 
23. Akhondi-Meybodi, M.; Ghane, M.; Akhondi-Meybodi, S.; Dashti, G. Five-year Survival Rate for Gastric Cancer in Yazd Province, Central Iran, from 2001 to 2008. Middle East J. Dig. Dis. 2017, 9, 39-48. [CrossRef] [PubMed]

24. Aziz, D.M. Assessment of bovine sperm viability by MTT reduction assay. Anim. Reprod. Sci. 2006, 92, 1-8. [CrossRef] [PubMed] 